Thayane Alves dos Santos

Rodrigues $^{1}$

(C) https://orcid.org/0000-0001-8457-6709

Lauane Pereira de Sousa Rodrigues

Ohttps://orid.org/0000-0003-1968-1967

Ângela Maria Rosas Cardoso²

Ohttps://orcid. org/0000-0002-3865-4320

\section{Adolescentes usuários de serviço de saúde mental: avaliação da percepção de melhora com o tratamento}

\author{
Adolescents users of mental health services: assessment of the perception \\ of improvement with treatment Assessment of perceived improvement
}

DOl: $10.1590 / 0047-2085000000269$

\section{RESUMO}

Objetivo: Avaliar a mudança percebida pelos adolescentes usuários de um serviço ambulatorial especializado de saúde mental infantojuvenil. Métodos: Estudo transversal com amostragem por conveniência. Foram realizadas entrevistas individuais com questionário que inclui questões demográficas e socioeconômicas, juntamente com a Escala de Mudança Percebida - versão paciente, validada no Brasil. Essa escala aborda mudanças percebidas em diversas dimensões da vida: ocupação, saúde física, aspectos psicobiológicos, sono, relacionamento e estabilidade emocional. O estudo foi realizado de agosto de 2017 a novembro de 2018, abrangendo 100 adolescentes de 12 a 18 anos em tratamento no serviço, no mínimo há 6 meses. Resultados: A maioria foi do sexo feminino (64,0\%), com idade entre 16 e 18 anos (48,0\%), atendida há mais de um ano (84,0\%). Os diagnósticos mais citados pelos adolescentes foram transtorno do déficit de atenção e hiperatividade (39\%) e transtornos de ansiedade (36\%). O resultado da avaliação global apontou o sentimento de melhora em 83\% dos participantes; quanto à mudança percebida nos itens da escala relacionados à melhora, incluem-se: interesse em trabalhar (71,0\%), convivência com a família $(67,0 \%)$ e interesse pela vida $(60,0 \%)$. Conclusão: A avaliação da assistência à saúde oferecida pelos serviços públicos de saúde mental é um importante indicador de qualidade e resolutividade das ações, possibilitando identificar os aspectos a serem aperfeiçoados ou reforçados nos processos de trabalho, a fim de favorecer melhores práticas de cuidado na infância e na adolescência.

\section{PALAVRAS-CHAVE}

Serviço de saúde mental, satisfação do paciente, avaliação em saúde, percepção, adolescente.

\section{ABSTRACT}

Objective: To evaluate the change perceived by adolescents, who are users of a specialized outpatient mental health service for children and adolescents. Methods: Cross-sectional study with convenience sampling. Individual interviews were conducted with a questionnaire that includes demographic and socioeconomic questions, together with the Perceived Change Scale - patient version, validated in Brazil. This scale addresses perceived changes in several dimensions of life: occupation, physical health, psychobiological aspects, sleep, relationship and emotional stability. The study was conducted from August 2017 to November 2018, covering 100 adolescents between 12 and 18 years undergoing treatment in the service for at least 6 months. Results: The majority were female (64.0\%), aged between 16 and 18 years (48.0\%), attended for more than one year (84.0\%). The most cited diagnoses by teenagers were attention deficit hyperactivity disorder (39\%) and anxiety disorders (36\%). The result of the global evaluation showed the feeling of improvement in $83 \%$ of the participants; regarding the perceived change in the items of the scale related to improvement, they include: interest in working (71.0\%), coexistence with family (67.0\%) and interest in life (60.0\%). Conclusion: The evaluation of health care offered by public mental health services is an important indicator of quality and resoluteness of actions, making it possible to identify the aspects to be improved or reinforced in work processes, in order to favor better care practices in childhood and adolescence.

\section{KEYWORDS}

Mental health service, patient satisfaction, health evaluation, perception, adolescent.

Received in: Mar/25/2020. Approved in: Apr/04//2020

1 Escola Superior de Ciências da Saúde (ESCS), Brasilia, DF, Brasil.

2 Escola Superior de Ciências da Saúde (ESCS); Universidade de Brasília (UnB), Faculdade de Ciências da Saúde, Programa de Pós-Graduação em Bioética, Brasilia, DF, Brasil.

Address for correspondence: Ângela Maria Rosas Cardoso. SGAS 605, Lotes 33/34, Asa Sul. 70200-650 - Brasília, DF, Brasil. E-mails: angelamrosas@hotmail.com; angelamrosas@gmail.com 


\section{INTRODUÇÃO}

A adolescência é considerada uma fase de aquisição de capacidades socioemocionais fundamentais para a construção de uma vida saudável, a partir do apoio e das condições de desenvolvimento proporcionadas pela família, escola e comunidade'. No entanto, também é considerada um período de grande vulnerabilidade, tendo em vista que os jovens se encontram expostos a inúmeras situações que podem aumentar o risco de surgimento de problemas de saúde mental $^{2-4}$. Segundo os autores, tais problemas podem persistir ao longo da vida adulta.

O estudo de metanálise realizado por Polanczyk et al. ${ }^{5}$ estima que a prevalência de transtornos mentais na infância e adolescência é de (13,4\%), sendo mais comuns os transtornos ansiosos (6,5\%), os transtornos depressivos (2,6\%), o transtorno do déficit de atenção e hiperatividade - TDAH $(3,4 \%)$, o transtorno opositor desafiante $(3,6 \%)$ e o transtorno de conduta (2,1\%). O estudo de coorte realizado no Brasil constatou a prevalência de 13,2\% de diagnósticos de transtornos mentais com início na adolescência, 15,6\% entre os meninos e $10,7 \%$ entre as meninas ${ }^{6}$. Nesse estudo, os autores observaram que os transtornos mais comuns foram os transtornos ansiosos (4,3\%), os transtornos depressivos (3,0\%), os transtornos do humor (1,2\%), o TDAH ou outra hiperatividade $(4,0 \%)$, sendo predominante o tipo combinado de TDAH, transtorno de conduta e TDO, de 2,8\%.

A atual política de Saúde Mental para Crianças e Adolescentes tem como proposta orientar-se pelos princípios do Estatuto da Criança e do Adolescente (ECA), da Lei no 10.216, de 2011, e dos marcos éticos da Reforma Psiquiátrica?. Nesse contexto, a organização dos serviços de atenção em saúde mental, como a Rede de Atenção Psicossocial (RAPS), foi instituída com a publicação da Portaria no 3.088, de 2011, do Ministério da Saúde (MS), com os objetivos de ampliar o acesso à atenção psicossocial, garantir a articulação de pontos de atenção à saúde, no âmbito do Sistema Único de Saúde (SUS), e implementar o cuidado em saúde integral com diferentes graus de complexidade -, favorecendo maior integração social, autonomia, protagonismo e participação dos seus usuários ${ }^{8}$.

$\mathrm{Na}$ especificidade da atenção à saúde mental na infância e adolescência, destacam-se os Centros de Atenção Psicossocial Infantojuvenil (CAPSi), cujas atividades devem ser voltadas para a inserção dessa população na família e na comunidade, incluindo o atendimento à família, atividades na comunidade com foco na integração da criança e do adolescente na família, na escola, na comunidade ou quaisquer outras formas de inserção social, o desenvolvimento de ações intersetoriais, de preferência nas áreas de assistência social, educação e justiça. Ressalta-se que a Portaria no 3.588, de 2017, incluiu como componente da RAPS os serviços ambulatoriais multiprofissionais de saúde mental, com o objetivo de ampliar e garantir o acesso à população?.

No entanto, ao longo da sua implementação, a política de saúde mental para crianças e adolescentes vem enfrentando problemas estruturais que requerem ações e investimentos consistentes para que sejam efetivamente superados, visando à consolidação da política no país. Entre esses problemas, podemos citar a dificuldade de expansão e distribuição regional conforme a faixa etária, as estratégias insuficientes de formação e capacitação dos profissionais, a dificuldade de articulação intersetorial e a ausência de estratégias de avaliação e monitoramento sintonizadas com os princípios e diretrizes do SUS ${ }^{10-12}$. Ainda, a fragmentação da assistência à saúde é uma prática assistencialista que reproduz o modelo de saúde biomédico, ou seja, centrado na medicalização e na exclusão dos sujeitos dos seus processos terapêuticos ${ }^{13}$.

A dificuldade de acesso aos serviços de saúde da RAPS infantojuvenil representa um importante prejuízo na detecção precoce de sintomas, constituindo um aspecto que aumenta a vulnerabilidade dessa população. Isso porque os diagnósticos de transtornos mentais e as intervenções precoces podem diminuir o sofrimento das famílias e favorecer melhores condições de desenvolvimento e aquisição de competências e prognóstico ao longo da vida adulta ${ }^{3}$.

Skokauskas et al. ${ }^{14}$ reforçam que a quantidade de serviços disponíveis para atender crianças e adolescentes e o número de profissionais de saúde, especialmente em serviços especializados de saúde mental, não acompanharam o aumento do número de casos de transtornos mentais na adolescência, apontando para a necessidade de reorientação dos serviços de saúde mental, incluindo a otimização de recursos existentes, o fortalecimento da atenção primária e as ações de promoção da saúde mental de crianças e adolescentes.

Nesse contexto, a avaliação dos serviços apresenta-se como um recurso para analisar a resolutividade das ações de cuidado, fortalecer as boas práticas e as ações de rede de saúde e intersetoriais, visando à ampliação do cuidado em saúde mental e ao desenvolvimento da criança e do adolescente. Entretanto, a maioria dos estudos disponíveis avalia a prevalência de transtornos ${ }^{5,6,15}$ ou diferentes intervenções e programas de tratamento ${ }^{16}$, sendo escassos os estudos de avaliação do tratamento em serviços ambulatoriais, especialmente os infantojuvenis. A avaliação de serviços pelos pacientes é compreendida como uma importante estratégia para a valorização do seu protagonismo no tratamento e na garantia de ações e cuidados mais resolutivos, tendo em vista que eles, os pacientes, são os mais afetados pelas fragilidades na atenção à saúde pelos profissionais e organização dos serviços ${ }^{17}$.

Portanto, considerando a inclusão do ambulatório especializado como um dispositivo da RAPS, este estudo visa avaliar a percepção de melhora dos adolescentes em 
tratamento em um serviço ambulatorial especializado de saúde mental infantojuvenil, a partir do uso da Escala de Mudança Percebida (EMP) - versão paciente.

\section{MÉTODOS}

Trata-se de um estudo quantitativo, transversal, prospectivo, realizado em um serviço especializado de Saúde Mental Infantojuvenil da Atenção Secundária, da Secretaria de Saúde do Distrito Federal.

A amostra de conveniência é composta por 100 adolescentes entre 12 e 18 anos, em tratamento no serviço há no mínimo seis meses. Os adolescentes foram abordados na sala de espera, por meio de convite para participação da pesquisa, no momento em que aguardavam as consultas.

Foi explicado para os adolescentes e pais ou responsáveis o objetivo da pesquisa, informando-Ihes que não havia obrigatoriedade em participar e nem a pesquisa causaria prejuízo no atendimento no serviço. Ao concordarem em participar, forneceu-se o termo de assentimento e consentimento e, após o seu preenchimento, procedeu-se à coleta de dados. Os instrumentos foram aplicados pelas pesquisadoras em um local protegido e individualizado.

A coleta de dados foi realizada entre agosto de 2017 e novembro de 2018, no período vespertino, às segundas, quartas e quintas-feiras, de acordo com a disponibilidade das pesquisadoras.

Conforme mencionado, o estudo foi realizado em um serviço especializado de saúde mental infantojuvenil da Atenção Secundária da Secretaria de Saúde do Distrito Federal, responsável por atender adolescentes de 12 anos até 17 anos, 11 meses e 29 dias, com transtornos mentais moderados ou uso eventual de substâncias psicoativas, na modalidade ambulatório. Esse serviço tem como objetivo atender integralmente à saúde do adolescente e sua família por meio de equipe multiprofissional e transdisciplinar, com base na Abordagem Biopsicossocial, de acordo com o referencial da Organização Mundial de Saúde, do MS e da Sociedade Brasileira de Pediatria.

Esse serviço também é supervisionado pela Diretoria de Serviços de Saúde Mental da Secretaria de Estado de Saúde (DISSAM/SESDF), responsável técnica pelos serviços públicos que prestam assistência especializada em saúde mental para os usuários do SUS, constituindo-se um componente da RAPS.

Para a coleta de dados, foi utilizada a EMP - versão paciente. A EMP ganhou destaque entre os instrumentos de medida, pois possibilita aos próprios pacientes avaliar as mudanças percebidas em diferentes dimensões da vida, em decorrência do tratamento recebido. Essa escala canadense foi adaptada para o Brasil por Bandeira et al. ${ }^{18}$. De modo geral, os estudos que utilizaram a EMP concluem pela eficácia da assistência por meio dos relatos de autopercepção de melhora dos usuários que responderam ao questionário, sendo esse o principal motivador para persistirem com o tratamento ${ }^{19}$.

Essa escala aborda mudanças percebidas pelos próprios pacientes em diversas dimensões de sua vida - como a biológica e social -, além de investigar os fatores associados à percepção de mudança. A versão brasileira da EMP possui 19 itens, sendo 18 para avaliar a percepção de mudança em aspectos gerais: Ocupação, Dimensão Psicológica, Relacionamentos e Saúde Física. Os aspectos se subdividem em alternativas de resposta dispostas em escala tipo Likert de três pontos, sendo: (1) Pior do que antes; (2) Sem mudança; (3) Melhor do que antes. O 190 item funciona como um indicador geral a ser respondido pelo paciente, conforme este se sente após iniciar o tratamento naquela instituição.

Também foi utilizado um questionário sociodemográfico, que incluía perguntas sobre escolaridade, número de reprovações, renda e recebimento de benefício social e questões relacionadas ao tratamento, como tempo de tratamento, tipos de serviços utilizados pelos adolescentes (hebiatria, neurologia, psiquiatria, terapia ocupacional, psicologia, ginecologia, nutrição, odontologia, programa de violência sexual, grupos terapêuticos ou práticas integrativas em saúde), diagnóstico referido pelo adolescente e a instituição que o encaminhou ao serviço.

Os dados coletados foram computados no programa SPSS (Statistical Package for Social Sciences), versão 13.0. Foi realizada análise descritiva simples das respostas, tanto das variáveis sociodemográficas quanto dos itens da EMP nas respostas de melhora, piora e ausência de mudança.

No que se refere à percepção de melhora relacionada ao tratamento recebido, a EMP possui um item classificado como aberto, no qual o entrevistado escolhe entre duas opções: "sim" - em caso de melhora percebida desde que iniciou o tratamento - e "não" - caso não tenha percebido mudança. Após a escolha, o entrevistado relata como o tratamento o ajudou a se sentir melhor.

Para análise dessas respostas, foi utilizado o software IRaMuTeQ, considerado uma ferramenta para o processamento de dados. O IRaMuTeQ tem como função a análise textual, cuja base é a classificação hierárquica descendente; entretanto, também inclui outras formas de análises, como a nuvem de palavras utilizada neste estudo. Essa ferramenta possibilita o agrupamento das palavras em nível significativo a partir da sua frequência no corpus do texto, sendo as palavras em maior destaque as estatisticamente com mais significância.

O estudo foi aprovado pelo Comitê de Ética em Pesquisa, da Fundação de Ensino e Pesquisa de Ciências da Saúde, sob o n’ 2.080.032, conforme a Resolução n 466/2012. 


\section{RESULTADOS}

Os resultados referentes às características sociodemográficas e clínicas dos pacientes estão dispostos na tabela 1. Destaca-se que a maioria é do sexo feminino (64,0\%), com idade média de 16 a 17 anos (46,0\%). Em relação à escolaridade, todos frequentavam a escola: $50 \%$ cursavam do quinto ao nono ano do ensino fundamental e $29 \%$ relataram ter reprovado pelo menos uma vez. Em relação à renda familiar, $45 \%$ apontaram que os pais recebem até dois salários mínimos e 53\% recebem algum tipo de benefício social, como passe livre estudantil.

Na questão aberta "motivo pelo qual está no serviço", alguns adolescentes não souberam relatar qual serviço os encaminhou, e classificamos como "outros" os adolescentes que citaram como motivo de encaminhamento o bullying, comportamento violento e agressivo, não saber lidar com a ansiedade, procura por medicamentos e tristeza. Entre os motivos, 30\% dos entrevistados relataram que foram encaminhados por algum serviço de saúde - da atenção pública ou privada -, 17\% procuraram o serviço por livre demanda e $17 \%$ foram encaminhados pela escola.

O diagnóstico mais prevalente foi o de TDAH (39\%), seguido de transtornos de ansiedade (36\%), transtornos depressivos (29\%) e violência sexual (9\%). Os participantes também citaram outros diagnósticos e motivos pelos quais estavam no serviço. Dentre os diagnósticos citados, destacaram-se distúrbio do sono, transtorno bipolar, distúrbio alimentar, epilepsia, surto psicótico, estresse pós-traumático e síndrome do pânico.

Quanto ao tempo de tratamento da amostra estudada, $80 \%$ encontravam-se em tratamento no serviço havia mais de um ano e $71 \%$ estavam sendo atendidos pela equipe multiprofissional (atendimento médico e de outros profissionais de saúde, como psicólogo, assistente social e enfermeiro).

Quanto à percepção de mudança pelo adolescente, relacionada ao tratamento, as respostas estão apresentadas na tabela 2. Cada item da EMP avaliou aspectos relacionados à ocupação e saúde física, aspectos psicológicos e sono e relacionamento e estabilidade emocional, respectivamente.

Na subescala ocupação e saúde física, nos itens capacidade de cumprir obrigações e tomar decisões e o interesse em trabalhar ou se ocupar com alguma coisa, houve percepção de melhora de $57 \%$ e $71 \%$, respectivamente. Ainda, nessa mesma subescala, o item sexualidade apresentou ausência de mudança (65\%). No item energia, 15\% relataram piora.

Os resultados em relação à avaliação da mudança percebida na subescala aspectos psicológicos e sono, os itens confiança em si, humor e suportar situações difíceis alcançaram $60 \%$, e interesse pela vida e problemas pessoais, $56 \%$. Quanto à subescala relacionamento e estabilidade emocional, houve relato de percepção de melhora na convivência familiar (67\%), enquanto estabilidade das emoções e convivência com os amigos apresentaram 59\%.
Tabela 1. Frequências absolutas (n) e relativas (\%) das variáveis sociodemográficas dos participantes - Brasília-DF, 2017-2018

\begin{tabular}{|c|c|c|}
\hline Variáveis & $\begin{array}{c}\text { Feminino } \\
\mathrm{n}=64\end{array}$ & $\begin{array}{c}\text { Masculino } \\
n=36\end{array}$ \\
\hline \multicolumn{3}{|l|}{ Idade do adolescente } \\
\hline $12-13$ & 11 & 6 \\
\hline $14-15$ & 22 & 13 \\
\hline $16-17$ & 31 & 15 \\
\hline 18 & 0 & 2 \\
\hline \multicolumn{3}{|l|}{ Escolaridade } \\
\hline $1^{\circ}$ ao $5^{\circ}$ ano do ensino fundamental I & 0 & 1 \\
\hline $6^{\circ}$ ao $9^{\circ}$ ano do ensino fundamental II & 34 & 16 \\
\hline Ensino médio & 30 & 19 \\
\hline \multicolumn{3}{|l|}{ Número de repetências } \\
\hline Não repetiu & 34 & 12 \\
\hline $1 \mathrm{vez}$ & 15 & 14 \\
\hline 2 vezes & 10 & 6 \\
\hline$>3$ vezes & 5 & 4 \\
\hline \multicolumn{3}{|l|}{ Profissão/ocupação/estágio } \\
\hline Estudante & 60 & 32 \\
\hline Estagiário & 4 & 4 \\
\hline \multicolumn{3}{|l|}{ Recebe benefício social } \\
\hline Bolsa família & 3 & 3 \\
\hline Passe livre urbano & 21 & 14 \\
\hline Benefício de prestação continuada & 1 & 0 \\
\hline Não recebe & 32 & 15 \\
\hline \multicolumn{3}{|l|}{ Renda familiar } \\
\hline Até 1 SM & 10 & 4 \\
\hline 1 a 2 SM & 20 & 11 \\
\hline 2 a 4 SM & 10 & 4 \\
\hline$>4 \mathrm{SM}$ & 6 & 2 \\
\hline Não sabe & 18 & 15 \\
\hline \multicolumn{3}{|l|}{ Tempo de tratamento } \\
\hline 6 meses a 1 ano & 10 & 6 \\
\hline 1 a 2 anos & 37 & 13 \\
\hline 2 a 3 anos & 7 & 4 \\
\hline Mais de 3 anos & 7 & 12 \\
\hline Não sabe & 3 & 1 \\
\hline \multicolumn{3}{|l|}{ Profissionais de saúde } \\
\hline Médico & 19 & 10 \\
\hline $\begin{array}{l}\text { Médico, enfermeiro, psicólogo, serviço } \\
\text { social, nutrição e odontologia }\end{array}$ & 45 & 26 \\
\hline \multicolumn{3}{|l|}{ Diagnóstico do adolescente } \\
\hline TDAH & 20 & 19 \\
\hline Transtornos depressivos & 24 & 5 \\
\hline Transtornos de ansiedade & 30 & 6 \\
\hline TOC & 2 & 2 \\
\hline Violência sexual & 9 & 0 \\
\hline Retardo mental & 1 & 3 \\
\hline Autismo & 0 & 1 \\
\hline Outros & 10 & 6 \\
\hline Não sabe & 4 & 0 \\
\hline \multicolumn{3}{|l|}{ Motivo pelo qual está no serviço } \\
\hline Encaminhamento escolar & 10 & 7 \\
\hline Encaminhamento de serviço de saúde & 19 & 11 \\
\hline Livre demanda & 12 & 5 \\
\hline Outros & 14 & 6 \\
\hline Não respondeu/não sabe & 9 & 7 \\
\hline
\end{tabular}

SM: salário mínimo; TDAH: transtorno do déficit de atenção e hiperatividade; TOC: transtorno obsessivo-compulsivo. 
Tabela 2. Percepção de mudança pelos pacientes, para a escala global e subescalas da Escala de Mudança Percebida (EMP) - versão paciente - Brasília-DF, 2017-2018

\begin{tabular}{|c|c|c|c|c|}
\hline Subescalas, item global e escala global & Itens & $\%$ Piora & \% Sem mudança & \% Melhora \\
\hline \multirow[t]{8}{*}{ Ocupação e saúde física } & Atividades de lazer & 8 & 37 & 55 \\
\hline & Energia & 15 & 42 & 43 \\
\hline & Tarefas de casa & 5 & 52 & 43 \\
\hline & Capacidade de cumprir obrigações e tomar decisões & 9 & 34 & 57 \\
\hline & Interesse em trabalhar ou se ocupar com alguma coisa & 3 & 26 & 71 \\
\hline & Sexualidade & 4 & 65 & 31 \\
\hline & Apetite & 13 & 42 & 43 \\
\hline & Saúde física & 13 & 35 & 52 \\
\hline \multicolumn{5}{|l|}{ Aspectos psicológicos e sono } \\
\hline & Sentimento de confiança em si próprio & 9 & 31 & 60 \\
\hline & Humor & 6 & 34 & 60 \\
\hline & Problemas pessoais & 4 & 40 & 56 \\
\hline & Sentimentos de interesse pela vida & 11 & 33 & 56 \\
\hline & Capacidade de suportar situações difíceis & 4 & 36 & 60 \\
\hline & Sono & 12 & 41 & 47 \\
\hline \multicolumn{5}{|l|}{ Relacionamento e estabilidade emocional } \\
\hline & Estabilidade das emoções & 9 & 32 & 59 \\
\hline & Convivência com a família & 3 & 30 & 67 \\
\hline & Convivência com amigos e amigas & 2 & 39 & 59 \\
\hline & Convivência com outras pessoas & 4 & 36 & 60 \\
\hline Item global & Avaliação global das mudanças & 2 & 15 & 83 \\
\hline
\end{tabular}

Por conseguinte, no item de avaliação global das mudanças, 83\% relataram que, de maneira geral, se sentiam melhor do que antes do início do tratamento.

Durante a avaliação pelo método da nuvem de palavras do item classificado como aberto, que se direcionava à sensação de melhora e quais fatores teriam ou não propiciando essa percepção, a palavra SIM foi a que apresentou mais repetições, seguida da palavra REMÉDIO. Constata-se na figura que as palavras mais frequentes aparecem em destaque, certificando o realce no corpus da pesquisa, conforme figura 1.



Figura 1. Nuvem de palavras - software IRAMUTEQ.

\section{DISCUSSÃO}

Segundo os resultados encontrados, é possível constatar que a maioria dos entrevistados relatou, no item de avaliação global da EMP, 83\% de melhora percebida desde que iniciou o tratamento. Entre os dados sociodemográficos, a maioria dos entrevistados é do sexo feminino, dado que corrobora com o estudo de Lopes et al. ${ }^{15}$, no qual evidenciaram a maior incidência de transtornos mentais em meninas quando comparadas com meninos da mesma faixa etária.

Em relação à renda familiar, $14 \%$ dos participantes relataram que a renda familiar é inferior a um salário mínimo e para 31\% ela é de um a dois salários mínimos. Esse dado aponta para a vulnerabilidade social das famílias atendidas no serviço, tendo em vista que a baixa renda também reduz a oportunidade de acesso aos serviços de saúde. Isso se deve à precariedade e à redução da oferta de saúde especializada em comunidades mais pobres, o que proporcionaria o diagnóstico precoce e intervenções para garantir o melhor desenvolvimento na infância20,21 .

Gomes e Santos ${ }^{22}$ ressaltam que os familiares de pacientes com sofrimento psíquico apresentam sofrimentos decorrentes de dificuldades financeiras, insegurança quanto ao futuro, sobrecarga em razão da persistência dos sintomas e agravamento do quadro de adoecimento em razão do 
acesso limitado à assistência à saúde mental. Os fracassos sociais, dificuldades de comunicação e interação produzem frustração e desesperança, aumentando a possibilidade de isolamento da vida comunitária, o estigma e a discriminação dessas pessoas ${ }^{23}$.

Esses aspectos apontam para a importância de articulação intersetorial no cuidado às crianças e adolescentes. Neste estudo, a escola é responsável por 17\% dos encaminhamentos para o serviço especializado, ressaltando-se o alto índice de reprovação entre os participantes, que alcançou 54\%; desses, 29,6\% relataram ter sido reprovados pelo menos duas vezes. Outro dado que chama a atenção é o fato de que $20 \%$ dos participantes citaram outros motivos pelos quais estavam no serviço, entre os quais o bullying, a violência física entre pares e a falta de amigos na escola.

Estudos apontam a grande demanda de acolhimentos nos CAPSi, devido a encaminhamentos das escolas para avaliação de problemas escolares ${ }^{24,25}$. Diante desse aspecto, Beltrame e Boarini ${ }^{24}$ ressaltam as dificuldades de articulação entre a educação e a saúde, revelando aspectos a serem discutidos para melhorar o cuidado a crianças e adolescentes, tendo em vista a associação entre as dificuldades escolares, problemas familiares e violências, com a necessidade de avaliação médica para tratamento em serviço de atenção especializada de saúde mental.

Nesse contexto, torna-se fundamental romper com a naturalização que permeia a demanda de encaminhamentos relacionados a queixas escolares e capacitar os profissionais envolvidos no cuidado à criança e ao adolescente, favorecendo uma compreensão mais ampla do seu contexto e necessidades, evitando, assim, o reducionismo das suas dificuldades e sofrimentos $24,26,27$.

A abordagem centrada no modelo biomédico e assistencialista também limita a atuação da clínica na Atenção Primária à Saúde a encaminhamentos para serviços especializados, especialistas e realização de exames, fortalecendo a lógica intervencionista ${ }^{13,28}$. Wenceslau e Ortega ${ }^{29}$ ressaltam, em sua análise teórica sobre a integração da saúde mental na atenção primária sob a perspectiva dos objetivos e estratégias da Saúde Mental Global (Global Mental Health - GMH), que o caminho para que as pessoas que sofrem com transtornos mentais tenham suas necessidades de cuidados atendidas só é possível por meio de uma atenção primária forte e integrada a uma rede de saúde mental organizada.

Portanto, reforça-se a importância de melhorar a articulação entre os serviços de atenção primária e os serviços de atenção especializada em saúde mental infantojuvenil, visando melhorar a qualidade da assistência a essa população e reduzir os prejuízos causados pela dificuldade de acesso e falta de resolutividade na assistência. É também importante que o tratamento medicamentoso não seja o único recurso a ser aplicado, dado que a criança e o adolescente devem ser vistos na sua integralidade, demandando uma abordagem biopsicossocial e intervenções não medicamentosas. Entretanto, na análise da nuvem do IRaMuTeQ, quanto aos motivos de melhora na questão aberta da EMP - versão paciente, a palavra "remédio" apareceu como importante fator citado pelos adolescentes como responsável pela melhora, o que reforça a visão biomédica de tratamento e a supervalorização medicamentosa como estratégia eficaz de melhora da população, aspecto também citado no estudo de Camatta et al. ${ }^{30}$.

Entre os diagnósticos mais citados pelos adolescentes, estão o TDAH (39\%) e o transtorno de ansiedade (36\%), o que corrobora dados de outros estudos. Segundo Dorneles et al. ${ }^{31}$, a prevalência mundial de TDAH é de cerca de 5,29\% em crianças e adolescentes; dos 39\% usuários identificados com TDAH, 51,28\% eram do sexo masculino. Moffitt et al. ${ }^{32}$ constataram que, na infância, a maior prevalência de TDAH é evidenciada em meninos, atestando o achado neste estudo. Lopes et al..$^{15}$ apontam que os transtornos de ansiedade são mais frequentes em meninas, concordando com o grupo estudado, em que, dos participantes identificados com transtorno de ansiedade e transtorno depressivo, 83,3\% e 68,96\% eram mulheres, respectivamente.

Quanto ao atendimento ambulatorial dos adolescentes participantes do estudo, podemos destacar que $71 \%$ dos entrevistados estavam em atendimento multiprofissional, o que corrobora os objetivos desse dispositivo na RAPS infantojuvenil, destacando-se a inclusão dos participantes do estudo e familiares em grupos terapêuticos interdisciplinares e/ou atendimentos individuais no serviço social, psicologia, terapia ocupacional e fonoaudiologia. Weber e Juruena ${ }^{33}$ também ressaltam que o atendimento exclusivamente biomédico é insuficiente para abordar toda a complexidade dos transtornos mentais, uma vez que o indivíduo que possui transtorno mental demandando uma terapêutica contínua necessita de diversas intervenções psicossociais voltadas à abordagem multiprofissional.

Nesse sentido, ressaltamos o resultado da análise da EMP - versão paciente, no subgrupo ocupação e saúde física, em que os itens interesse em trabalhar ou se ocupar com alguma coisa, capacidade de cumprir obrigações e tomar decisões e atividade de lazer foram os que mais obtiveram percepção de melhora pelos adolescentes, o que pode indicar que o tratamento oferecido pelo serviço atue no fortalecimento do adolescente como sujeito de sua vida, proporcionando a tomada de decisão e a busca de atividades que sejam adequadas a suas preferências. No subgrupo relacionamento e estabilidade emocional, a convivência com a família e a convivência com outras pessoas foram os itens que mais obtiveram percepção de melhora pelos adolescentes, apontando para a importância da inclusão da família no serviço para o fortalecimento das relações. Nessa linha, 
estudos apontam que os transtornos citados pelos adolescentes trazem prejuízos nas relações familiares e sobrecarga de cuidado à família ${ }^{34,35}$.

Esses aspectos de percepção de melhora podem aumentar a capacidade de enfrentamento diante das adversidades na adolescência e reduzir o impacto dos transtornos mentais ao longo da vida, configurando-se como fator de proteção. Weinberg et al. ${ }^{36}$ ressaltam a satisfação com a vida e a melhor condição socioeconômica como fatores de proteção ao desenvolvimento de transtornos mentais.

A identificação de aspectos relacionados à percepção de melhora dos usuários de saúde mental acerca do tratamento, objetivo deste estudo, poderá contribuir para a avaliação da assistência à saúde e a resolutividade das ações e propostas para a redução do sofrimento psíquico, fortalecimento da autonomia e enfrentamento das adversidades dos adolescentes ao longo da vida. Ressalta-se que o desempenho da assistência em saúde deve atender à perspectiva dos usuários ${ }^{19}$.

\section{CONCLUSÃO}

Os itens referentes a relacionamento e estabilidade emocional foram os mais importantes quanto à percepção de meIhora, com destaque para o avanço percebido na convivência em sociedade e família. O interesse em trabalhar ganhou evidência entre os itens das subescalas como o de maior evolução, reforçando a importância do tratamento para a autonomia e protagonismo do adolescente. Apesar de a maioria dos entrevistados estar em atendimento multiprofissional e interdisciplinar, o modelo biomédico continua ganhando destaque na perspectiva social, enfatizando a necessidade de desconstrução da visão de que apenas a intervenção farmacológica é benéfica.

A baixa cobertura de assistência à saúde mental na infância e na adolescência reforça a importância da avaliação dos serviços já implantados, tendo em vista que a avaliação da assistência oferecida pelos serviços de saúde é um importante indicador de qualidade e resolutividade das ações, oportunizando identificar os aspectos a serem aperfeiçoados ou reforçados, a fim de oferecer melhores práticas de cuidado e qualidade de vida para os pacientes. Por conseguinte, a percepção de melhora com o tratamento pelos pacientes constitui-se elemento relevante de avaliação para os profissionais de saúde mental e de saúde pública, oportunizando seu redirecionamento para as necessidades referidas pelos pacientes como ausentes de mudança, sendo, portanto, atributo para validação clínica e potencializando os serviços de saúde na sociedade.

Conforme proposto neste estudo, apresentamos uma contribuição para a área de saúde pública, estimulando a reavaliação do serviço prestado, com a finalidade de fornecer assistência adequada e individualizada aos diferentes usuários do SUS, garantindo o princípio da equidade, além de identificar os aspectos a serem aperfeiçoados ou reforçados nos serviços de saúde mental. Dessa forma, as informações levantadas podem contribuir para o redimensionamento do tratamento, de forma a garantir a assistência adequada às demandas e necessidades dos pacientes.

A análise dos dados deste estudo encontra limites na literatura, relacionados à dificuldade de comparação de dados devido à pouca literatura disponível quanto à avaliação de serviços na adolescência, o que aponta para a necessidade de mais estudos que propiciem a avaliação das atividades desenvolvidas a partir da perspectiva dos usuários nos diversos dispositivos da RAPS, especialmente infantojuvenil. Outras limitações incluem o tamanho da amostra, a ausência da avaliação dos medicamentos em uso e da sua regularidade e, ainda, a dificuldade de compreensão de alguns itens da escala pelos adolescentes, como sexualidade e humor. Esses aspectos apontam para a importância de validação de escalas para essa faixa etária e a importância de estudos futuros de avaliação de serviços feita pelos próprios adolescentes, para o fortalecimento da autonomia no tratamento, o que proporcionaria o melhoramento dos serviços de saúde mental, o que ressalta a importância deste estudo.

\section{CONTRIBUIÇÕES INDIVIDUAIS}

\section{Thayane Alves dos Santos Rodrigues e Lauane Pereira de Sousa Rodrigues - Participaram do delineamento do estudo e do trabalho de campo, analisaram os dados coleta- dos, interpretaram os resultados e foram responsáveis pela redação do manuscrito.}

Angela Maria Rosas Cardoso - Contribuiu com a supervisão do trabalho, desde sua concepção até sua finalização e revisão crítica do conteúdo do manuscrito.

Todos os autores aprovaram a versão final e são responsáveis por todos os aspectos do trabalho, incluindo a garantia de sua precisão e integridade.

\section{CONFLITO DE INTERESSES}

Não há conflito de interesses.

\section{REFERÊNCIAS}

1. World Health Organization (WHO). Adolescent mental health. Geneva; 2018. Disponível em: https://www.who.int/news-room/fact-sheets/detail/adolescent-mental-health. Acessado em: 21 mar. 2020.

2. Matos MB, Cruz ACN, Dumith SC, Dias NC, Carret RBP, Quevedo LA. Eventos estressores na família e indicativos de problemas de saúde mental em crianças com idade escolar. Ciênc Saúde Colet. 2015;20(7):2157-63. 
3. Collishaw S. Annual research review: Secular trends in child and adolescent mental health. J Child Psychol Psychiatry. 2015;56(3):370-93.

4. Danese A. Annual research review: Rethinking childhood trauma - new research directions for measurement, study design and analytical strategies. J Child Psychol Psychiatry. 2020;61(3):236-50.

5. Polanczyk GV, Salum GA, Sugaya LS, Caye A, Rohde LA. Annual Research Review: A metaanalysis of the worldwide prevalence of mental disorders in children and adolescents. J Child Psychol Psychiatry. 2015;56(3):345-65.

6. Maison CL, Munhos TN, Santos IS, Anselmi L, Barros FC, Matijasevich A. Prevalence and risk factors of psychiatric disorders in early adolescence: 2004 Pelotas (Brazil) birth cohort. Soc Psychiatry Psychiatr Epidemiol. 2018;53(7):685-97.

7. Braga CP, D'Oliveira AFPL. Políticas públicas na atenção à saúde mental de crianças e adolescentes: percurso histórico e caminhos de participação. Ciênc Saúde Colet. 2019;24(2):401-10.

8. Brasil. Ministério da Saúde. Gabinete do Ministro. Portaria nº 3.088, de 23 de dezembro de 2011. Instituiu a Rede de Atenção Psicossocial para pessoas com sofrimento ou transtorno mental e com necessidades decorrentes do uso de crack, álcool e outras drogas, no âmbito do Sistema Único de Saúde (SUS). Diário Oficial da União, seção 1, 2011.

9. Brasil. Ministério da Saúde. Gabinete do Ministro. Portaria n 3.588, de 21 de dezembro de 2017. Altera as Portarias de Consolidação no 3 e no 6, de 28 de setembro de 2017, para dispor sobre a Rede de Atenção Psicossocial, e dá outras providências. Diário Oficial da União, seção 1, p. 23-36, 2017.

10. Couto MCV, Delgado PGG. Crianças e adolescentes na agenda política da saúde mental brasileira: inclusão tardia, desafios atuais. Psicol Clin. 2015;27(1):17-40.

11. Costa PHA, Colugnati FAB, Ronzani, TM. Avaliação de serviços em saúde mental no Brasil: revisão sistemática da literatura. Ciênc Saúde Colet. 2015;20(10):3243-53.

12. Garcia CYC, Santos DN, Machado DB. Centros de Atenção Psicossocial Infantojuvenil no Brasil: distribuição geográfica e perfil dos usuários. Cad Saúde Pública. 2015;31(12):2649-54.

13. Tonin CF, Barbosa TM. A interface entre saúde mental e vulnerabilidade social. Tempus. 2018;11(3):50-68.

14. Skokauskas N, Fung D, Flaherty LT, von Klitzing K, Püras D, Servili C, et al. Shaping the future of child and adolescent psychiatry. Child Adolesc Psychiatry Mental Health. 2019;13:19.

15. Lopes CS, Abreu GA, Santos DF, Menezes PM, Carvalho KMB, Cunha CF, et al. ERICA: prevalência de transtornos mentais comuns em adolescentes brasileiros. Rev Saúde Pública. 2016;50(Supl 1):12-24.

16. Cleverley K, Rowland E, Bennett K, Jeffs L, Gore D. Identifying core components and indicators of successful transitions from child to adult mental health services: a scoping review. Eur Child Adolesc Psychiatry. 2020;29(2):107-21.

17. World Health Organization (WHO). Comprehensive mental health action plan 2013-2020. Geneva; 2013. Disponível em: https://apps.who.int/iris/bitstream/ handle/10665/89966/9789241506021_eng.pdf;jsessionid=23470431A5B5FFABC7ACEB7 DB6564699? sequence=1/. Acesso em: 21 mar. 2020.

18. Bandeira $M B$, Andrade $M C R$, Costa $C S$, Silva MA. Percepção dos pacientes sobre o tratamento em serviços de saúde mental: validação da Escala de Mudança Percebida. Psicol Reflex Crít. 2011;24(2):236-44.
19. Cardoso LS, Siqueira MM, Vieira, CB. Avaliação da satisfação e percepção de mudanças entre usuários de um programa de alcoolismo. Rev Bras Pesqui Saúde. 2016;18(1):87-95.

20. Silva DF, Santana PR. Transtornos mentais e pobreza no Brasil: uma revisão sistemática. Tempus. 2012;6(4):175-85.

21. Cini RA, Rosaneli CF, Sganzerla A. Categorização dos sujeitos em condição de vulnerabilidade: uma revisão na perspectiva da bioética. Rev Iberoam Bioética. 2017;5:1-16.

22. Gomes TB, Santos JBF. Dilemas e vicissitudes de famílias em situação de vulnerabilidade social no contexto da desinstitucionalização psiquiátrica. Physis Rev Saúde Coletiva. 2016;26(1):271-87.

23. Trombetta F, Ramos RS, Misiak M. Familiares de portadores de doença mental: Expectativas sobre a doença. Cad Bras Saúde Mental. 2015;7(16):62-71.

24. Beltrame MM, Boarini ML. Saúde mental e infância: reflexões sobre a demanda escolar de um CAPSi. Rev Psicol Ciênc Prof. 2013;33(2):336-49.

25. Viégas $L S$, Freire KES, Bomfim FB. Atendimento a queixa escolar nos serviços públicos de saúde mental da Bahia. Rev Psicol Esc Educ. 2018;22(1):133-40.

26. Azevedo LJC. Medicalização das infâncias: entre os cuidados e os medicamentos. Psicologia USP. 2018;29(1):451-8.

27. Lemos FCS, Galdino D, Rodrigues RD. Processos de Medicalização de Crianças e Adolescentes nos relatórios da UNICEF. Pesqui Prát Psicossociais. 2014;9(2):201-12.

28. Filho JAS, Bezerra AM. Acolhimento em Saúde Mental na Atenção Primária à Saúde: revisão integrativa. Revista Multidisciplinar e de Psicologia. 2018;12(40):613-27.

29. Wenceslau LD, Ortega F. Salud mental en la atención primaria y Salud Mental Global: perspectivas internacionales y el escenario brasileño. Interface (Botucatu). 2015;19(55):1121-32.

30. Camatta MW, Tocantins FR, Schneider JF. Ações de saúde mental na Estratégia Saúde da Família: expectativas de familiares. Rev Esc Anna Nery. 2016;20(2):281-8.

31. Dorneles BV, Corso VL, Costa AC, Pisaco NMT, Sperafico YLS, Rohde LAP. Impacto do DSM-5 no diagnóstico de Transtornos de Aprendizagem em Crianças e Adolescentes com TDAH: um estudo de prevalência. Psicol Reflex Crít. 2014;27(4):759-67.

32. Moffitt TE, Houts R, Asherson P, Belsky DW, Corcoran DL, Hammerle M, et al. Is adult ADHD a Childhood-Onset Neurodevelopmental Disorder? Evidence From a Four-Decade Longitudinal Cohort Study. Am J Psychiatry. 2015;172(10):967-77.

33. Weber CAT, Juruena MF. Paradigmas de atenção e estigma da doença mental na reforma psiquiátrica brasileira. Psic Saúde Doenças. 2017;18(3):640-59.

34. Vicente JB, Marconi SS, Higarashi, IH. Convivendo com o transtorno mental na infância: sentimentos e reações da família. Texto Contexto Enferm. 2016;25(1):e0370014.

35. Eloi SC, Oliveira EM, Lopes MVO, Parente JRF, Eloi SMC, Lima DS. Sobrecarga de cuidadores familiares de pessoas com transtornos mentais: análise dos serviços de saúde. Ciênc Saúde Coletiva. 2018;23(9):3001-11.

36. Weinberg D, Stevens GWJM, Duinho EL, Finkenauer C. Adolescent Socioeconomic Status and Mental Health Inequalities in the Netherlands, 2001-2017. Int J Environ Res Public Health. 2019;16(19). pii: E3605. 